Z. Epileptol. 2019 · 32:150-151 https://doi.org/10.1007/s10309-019-0250-1 Online publiziert: 2. April 2019

(c) Springer Medizin Verlag GmbH, ein Teil von Springer Nature 2019

Ein unkritischer Herstellerwechsel von Antikonvulsiva kann für Patienten mit Epilepsie weitreichende, negative Folgen i. S. von wieder oder vermehrt auftretenden epileptischen Anfällen haben.

Die Evidenz wird allerdings größer, dass Schwankungen in der Bioverfügbarkeit und somit der Blutspiegel zwischen den einzelnen Präparaten unterschiedlicher Hersteller dafür nicht ohne Weiteres herangezogen werden können - zumindest dann nicht, wenn Daten aus streng reglementierten Studien zugrunde gelegt werden $[1,7]$.

Es hat sich aber in großen Untersuchungen in der praktischen Wirklichkeit gezeigt, dass unter laufender Therapie Herstellerwechsel (insbesondere bei Änderungen des Aussehens der Tabletten) $\mathrm{zu}$ einer geringeren Adhärenz und bei anfallsfreien Patienten somit $\mathrm{zu}$ einem erhöhten Rezidivrisiko führen [2, 5, 6]. Das Risiko scheint bei älteren Patienten am größten zu sein [6]. Herstellerwechsel können zu einer Verunsicherung bei Patienten führen, die Einnahmefehler und dadurch eine suboptimale Therapie nach sich ziehen.

Mit der seitens der Deutschen Gesellschaft für Epileptologie e.V. (DGfE) insbesondere bei anfallsfreien Patienten empfohlenen Markierung des „Aut idem“-Feldes durch die behandelnden Ärzte soll vermieden werden, dass durch einen und im klinischen Alltag häufig

Aus Gründen der Lesbarkeit haben wir darauf verzichtet, geschlechtsspezifische Formulierungen zu verwenden. Soweit personenbezogene Bezeichnungen nur in männlicher Form angeführt sind, beziehen sie sich auf Frauen und Männeringleicher Weise.

Hajo Hamer ${ }^{1}$ Martin Holtkamp ${ }^{1}$ Thomas Mayer ${ }^{1}$ für Deutsche Gesellschaft für Epileptologie e.V.

${ }^{1}$ Deutsche Gesellschaft für Epileptologie e. V., Berlin, Deutschland

\title{
Stellungnahme zum Herstellerwechsel bei Antikonvulsiva
}

sogar mehrere Herstellerwechsel von Antikonvulsiva das oft mühevoll erreichte Therapieziel einer dauerhaften Anfalls- und/oder Nebenwirkungsfreiheit gefährdet wird. Bei diesen Patienten sollte die erfolgreiche Arzneimittelzubereitung, sei es ein Originalpräparat oder ein Generikum, in der Regel konstant eingesetzt werden, um eine verlässliche Behandlungssituation zu gewährleisten [3]. Bekanntermaßen können die Konsequenzen eines Rezidivanfalls gerade bei längerer Anfallsfreiheit bezüglich der Fahreignung, des Berufslebens etc. so einschneidend sein, dass möglichst jegliche Änderung bzgl. Hersteller und Präparaten vermieden werden muss, um dieses vornehmliche Therapieziel nicht zu kompromittieren.

Die insbesondere durch Rabattverträge nicht mehr kontrollierbare Abgabe von unterschiedlichen Generika eines gleichen Wirkstoffs ist somit für die Epileptologie nicht geeignet. Die Aufzahlung für Patienten bei „Aut idem“ angekreuzten Rezepten von Medikamenten mit Festpreis kann zu erheblichen finanziellen Belastungen (auch der zuzahlungsbefreiten Patienten) führen, was aus Sicht der Fachgesellschaft ebenfalls nicht akzeptabel ist und zu Umstellungen auf andere Festpreisprodukte führen kann. Die Entscheidung über einen Austausch muss in jedem Fall kritisch fachärztlich geprüft werden und ist somit den behandelnden Fachärzten zu überlassen. Nach Meinung der DGfE sollte bei Patienten mit einer guten individualisierten Einstellung weder leichtfertig noch aus kurzfristigen, rein ökonomischen Gründen eine Umstellung auf ein wirkstoff- gleiches Präparat mit anderer pharmazeutischer Formulierung oder Aussehen erfolgen. Auch sind im ambulanten Bereich Umstellungen zwischen verschiedenen Generika des gleichen Wirkstoffs aus ökonomischen Gründen in der Regel häufig nicht notwendig, da die Preisunterschiede minimal sind. Im stationären Bereich sind Antikonvulsiva oft nicht in allen generischen Versionen verfügbar. Hier ist das Risiko einer eingeschränkten Adhärenz durch Herstellerwechsel aufseiten der Patienten durch eine kontrollierte Tabletteneinnahme jedoch deutlich geringer als im ambulanten Setting. Bei einem passageren Herstellerwechsel im stationären Rahmen sollten den Patienten der Hintergrund und die pharmakologische Gleichwertigkeit der Präparate erklärt werden. Bei Neueinstellungen oder ohnehin erforderlichen Umstellungen der antiepileptischen Therapie können aus ökonomischen Gründen - sofern verfügbar - von vornherein kostengünstige Präparate eingesetzt werden [4].

\section{Zusammenfassung der praktischen Vorschläge}

1. Ein Herstellerwechsel sollte - insbesondere bei anfallsfreien Patienten möglichst vermieden werden.

2. Nach heutigem Wissensstand ist davon auszugehen, dass ein Herstelleroder Präparatewechsel die Adhärenz reduzieren kann.

3. Wenn ein Herstellerwechsel notwendig ist, sollten die Patienten von dem behandelnden Facharzt darüber aufgeklärt werden, dass allein eine Veränderung der Erscheinungsform 
der Tablette oder der Verpackung keine pharmakologischen Auswirkungen hat.

4. Kontrollen des Blutspiegels vor und nach einem Herstellerwechsel können sinnvoll sein, um die Adhärenz zu überprüfen.

5. Im stationären Setting sollten die $\mathrm{Pa}$ tienten über oft notwendige passagere Herstellerwechsel und deren nach jetziger Datenlage unbedenklichen pharmakologischen Auswirkungen aufgeklärt werden.

6. Bei Neueinstellungen oder erforderlichen Umstellungen der antiepileptischen Therapie sollten aus ökonomischen Gründen von vornherein kostengünstige Präparate eingesetzt werden.

\section{Korrespondenzadresse}

\section{Prof. Dr. med. Hajo Hamer, MHBA}

Deutsche Gesellschaft für Epileptologie e.V. Reinhardtstr. 27 c, 10117 Berlin, Deutschland ize@dgfe.info

\section{Einhaltung ethischer Richtlinien}

Interessenkonflikt. H.M. Hamer hat Honorare für Referate oder Manuskripte und/oder Unterstützungen für Fortbildungsveranstaltungen erhalten von Amgen, Ad-Tech, Bial, Bracco, Desitin, Eisai, IQWiG, Hexal, NeuroConsil, Nihon Kohden, Novartis, Pfizer, Thieme und UCB Pharma. Darüber hinaus hat er für Beratungen der Firmen Bial, Desitin, Eisai, GW, Novartis und UCB Pharma Honorare erhalten. M. Holtkamp hat in den letzten 3 Jahren für Vorträge und Beratungstätigkeiten von Bial, Desitin, Eisai, GW Pharmaceuticals, Medtronic, Novartis und UCB Pharma Honorare erhalten. T. Mayer hat in den letzten 3 Jahren für Vorträge und Beratungstätigkeiten von Bial, Desitin, Eisai, GW Pharmaceuticals, Zogenix, Novartis und UCB Pharma Honorare erhalten.

Dieser Beitrag beinhaltet keine von den Autoren durchgeführten Studien an Menschen oder Tieren.

\section{Literatur}

1. Berg M, Welty TE, Gidal BE, Diaz FJ, Krebill R, Szaflarski JP, Dworetzky BA, Pollard JR, Elder EJ Jr., Jiang W, Jiang X, Switzer RD, Privitera MD (2017) Bioequivalence between generic and branded lamotrigine in people with epilepsy: the EQUIGEN randomized clinical trial. JAMA Neurol 74:919-926

2. Gollwitzer S, Kostev K, Hagge M, Lang J, Graf W, Hamer HM (2016) Nonadherence to antiepileptic drugs in Germany: a retrospective, populationbased study. Baillieres Clin Neurol 87:466-472
3. Hensler K, Uhlmann C, Porschen T, Benecke R, Rosche J (2013) Generic substitution of antiepileptic drugs - a survey of patients' perspectives in Germany and other German-speaking countries. Epilepsy Behav 27:135-139

4. Holtkamp M, Theodore WH (2018) Generic antiepileptic drugs. Safe or harmful in patients with epilepsy? Epilepsia 59:1273-1281

5. Kesselheim AS, Misono AS, Shrank WH, Greene JA, Doherty M, Avorn J, Choudhry NK (2013) Variations in pill appearance of antiepileptic drugs and the risk of nonadherence. JAMA Intern Med 173:202-208

6. Lang JD, Kostev K, Onugoren MD, Gollwitzer S, Graf W, Muller T, Olmes DG, Hamer HM (2018) Switching the manufacturer of antiepileptic drugs is associated with higher risk of seizures: a nationwide study of prescription data in Germany. Ann Neurol 84:918-925

7. Privitera MD, Welty TE, Gidal BE, Diaz FJ, Krebill $R_{\iota}$ Szaflarski JP, Dworetzky BA, Pollard JR, Elder EJ Jr., Jiang W, Jiang X, Berg M (2016) Generic-to-generic lamotrigine switches in people with epilepsy: the randomised controlled EQUIGEN trial. Lancet Neurol 15:365-372 